\title{
Evaluating Adhesion Layers for Plasmonic Nanostructures with Monochromated STEM-EELS and Surface Enhanced Raman Spectroscopy
}

\author{
Steven J Madsen ${ }^{1}$, Ai Leen $\mathrm{Koh}^{2}$ and Robert Sinclair ${ }^{1}$ \\ ${ }^{1}$ Stanford University, Department of Materials Science and Engineering, Stanford, CA 94305-4034 USA \\ ${ }^{2}$ Stanford Nano Shared Facilities, Stanford University, Stanford, California 94305-4045, USA
}

Nano-plasmonics is a rapidly growing field of study with applications in energy, healthcare and security. Plasmonic structures frequently consist of noble metals deposited onto a substrate in a 'top-down' synthesis. This method requires an adhesion layer, often $\mathrm{Cr}$ or $\mathrm{Ti}$, to bind the optically active metal to the substrate. Recent publications have demonstrated the presence of these adhesion layers cause damping of plasmon resonances, observed by optical scattering measurements [1]. Because Raman surface enhancement relies on plasmon resonances near the laser excitation wavelength, these layers can cause reduction in surface enhanced Raman spectroscopy (SERS) intensity [2]. The present study uses monochromated electron energy-loss spectroscopy in a scanning transmission electron microscope (STEM-EELS) to compare plasmon resonances of structures with and without commonly used adhesion layers at the nanometer scale. Raman spectra were collected for the same structures, and variation in SERS enhancement factors correlate with the changes observed in plasmon intensity.

Nanopatterning was achieved by electron beam lithography of polymethyl methacrylate resist on either $\mathrm{Si}$ (for Raman) or thin SiN windows (for TEM). Samples were created with no adhesion layer, $2 \mathrm{~nm}$ of $\mathrm{Cr}$ or Ti, or vapor deposited mercaptopropyltrimethoxysilane (MPTMS). 30nm of gold, the plasmonic and Raman surface enhancing material, is evaporated on top of the patterned resist and adhesion layer (if present). To avoid film delamination, the resist was not lifted off, leaving a structure shown schematically in cross section in Fig. 1. Fig. 2 shows a plan view STEM dark field image of a typical sample.

STEM-EELS data, collected in a monochromated Titan at $300 \mathrm{kV}$, show the presence of several plasmon resonances in the energy range $1.3-2.4 \mathrm{eV}$, the expected range for gold nanostructures [3]. Fig. 3 shows EEL spectra collected from the marked regions in the inset. The samples with no adhesion layer and with MPTMS are similar, while both $\mathrm{Ti}$ and $\mathrm{Cr}$ result in dramatically reduced peak intensities. Lower energy localized surface plasmon resonance (LSPR) peaks are observed in the $1.5-1.7 \mathrm{eV}$ range at the marked location (Fig. 4). Here, gold with no adhesion layer shows the strongest peak, followed by MPTMS with the two metal underlayers $\mathrm{Cr}$ and Ti showing the weakest peaks.

Samples were coated with a self-assembled monolayer of 4-mercaptopyridine dye and tested using Raman spectroscopy at $785 \mathrm{~nm}$ and $532 \mathrm{~nm}$. Although there are large peaks observed in the $2.0-2.4 \mathrm{eV}$ range (Fig. 3), the excitation location has local rotation symmetry, which has been associated with 'dark' plasmon modes not accessible with photons [4]. The Raman spectra using a 532nm (2.33eV) laser confirm that there is not strong SERS enhancement for this energy (Fig. 5). By exciting with a 785nm $(1.58 \mathrm{eV})$ source near the LSPR energy observed in Fig. 4, Raman signal strengths orders of magnitude greater than those from the $532 \mathrm{~nm}$ laser can be observed. The SERS enhancement factors for a $1.58 \mathrm{eV}$ laser have the same trend as the EELS LSPR peak intensity at the same energy (Figs. 6,4) - the noadhesion-layer sample is greatest, then MPTMS, with $\mathrm{Cr}$ and Ti far lower.

In conclusion, both $\mathrm{Cr}$ and $\mathrm{Ti}$ cause significant plasmon damping which was detected using STEM- 
EELS. These layers are also shown to reduce SERS enhancement factor, which is expected to be a direct result of reduced plasmon resonance. MPTMS causes less damping and is thus a more desirable option.

[1] TG Habteyes et al, ACS Nano 6 (2012), p. 5702-5709.

[2] T Siegfried et al, ACS Nano 7 (2013), p. 2751-2757.

[3] FP Schmidt et al, Nano Letters 12 (2012), p. 5780-5783.

[4] AL Koh, et al, Nano Letters 11 (2011), p. 1323-1330.

[5] This research is supported by the Center for Cancer Nanotechnology Excellence and Translation (CCNE-T) grant funded by NCI-NIH to Stanford University U54CA151459.

$\quad 30 \mathrm{~nm} \mathrm{Au} \ll$ Adhesion Layer
PMMA resist
Substrate

Figure 1: Cross section schematic of fabricated structures. Samples were generated with no adhesion layer, $2 \mathrm{~nm} \mathrm{Ti}, 2 \mathrm{~nm} \mathrm{Cr}$, or vapor deposited MPTMS.

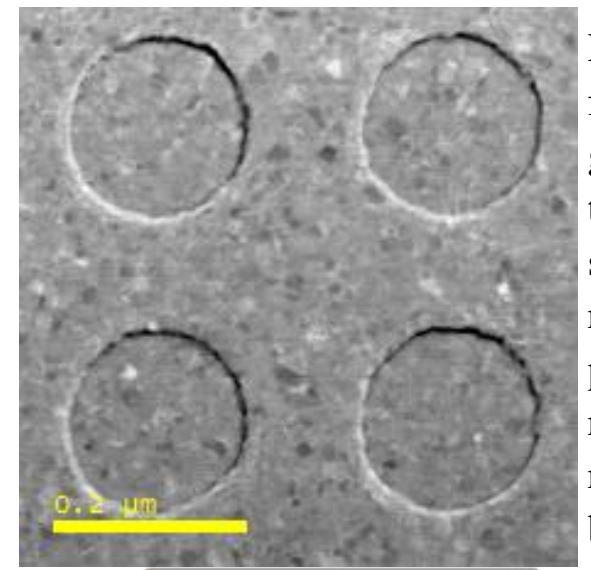

Figure 2 (left): Dark field STEM micrograph of a representtative gold nanostructure. The circular regions are gold nanoparticles depressed relative to the surrounding film. Scale bar 200nm.

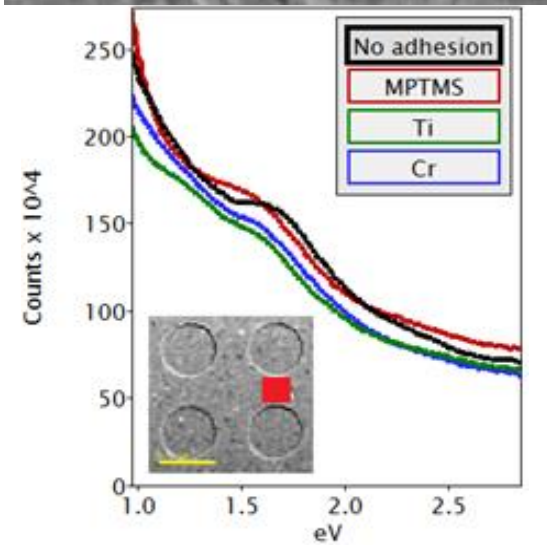

Figure 4: EEL spectra from the location indicated in inset. The sample without adhesion shows the strongest peak, and MPTMS is the least damping of the adhesion layers.

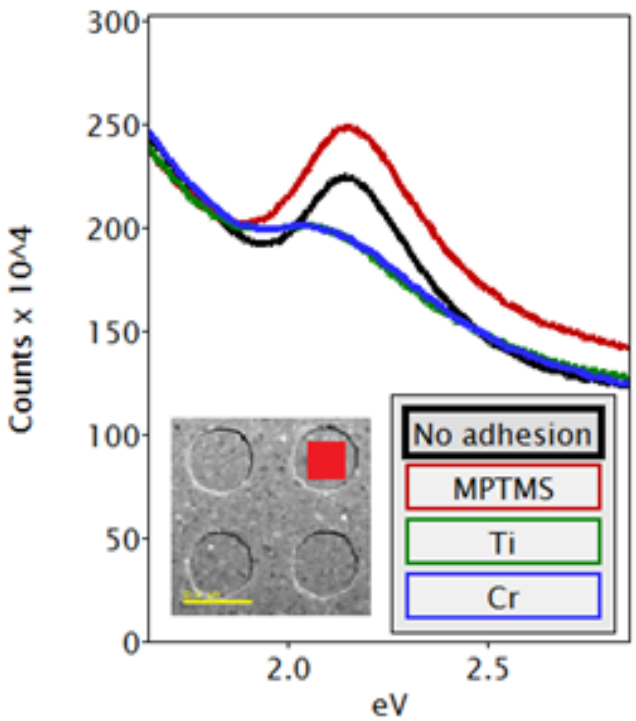

Figure 3: Low-loss EEL spectra comparing plasmon resonances observed with different adhesion layers. Inset: location from which EELS data was collected marked in red.

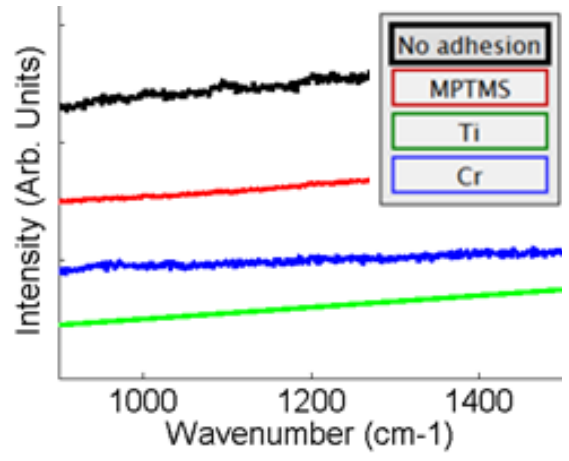

Figure 5: Raman data collected from samples coated with 4-MP dye using a $533 \mathrm{~nm}$ laser. SERS signal from the dye is not observed at this energy. Vertical axis offset for clarity.

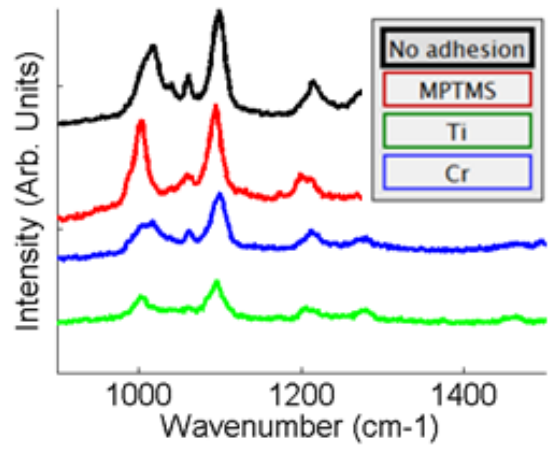

Figure 6: Raman data collected using a $785 \mathrm{~nm}$ laser. The trend in signal enhancement matches the trend in plasmon resonance observed at the excitation energy via STEM-EELS. Vertical axis offset for clarity. 